\title{
Factors Contributing to Intergenerational Sexual Relationships among Refugee Girls in the City of Tshwane, South Africa
}

\author{
Dr Azwihangwisi Helen Mavhandu-Mudzusi \\ Professor Peter Thomas Sandy \\ Tshabangu Kalala Francis
}

University of South Africa, Department of Health Studies, Preller Street, Pretoria, South Africa 0003

Email:mmudza@unisa.ac.za

\section{Doi:10.5901/mjss.2014.v5n20p2876}

\section{Abstract}

\begin{abstract}
Background::Young refugee girls are engaged in sexual relationships with older men under the guise of the phenomenon "sugar-daddy" with the primary motive of obtaining economic favours. Aim: This paper reports on a study that explored factors contributing to young refugee girls (aged 15-19 years) becoming engaged in intergenerational sexual relationships in the City of Tshwane, South Africa. Method: Interpretative phenomenological analysis was used in this study for both data collection and analysis. A total of 20 refugee girls living in the City of Tshwane were purposively selected to participate in the study. Data was collected through in-depth individual interviews. Results:: Powerlessness; increased libido; curiosity; perception of inferior status of the women; attitudes towards assertive women; culture; anonymity; high cost of living; unemployment; poor educational status and residential conditions emerged as the main contributing factors to the involvement of young refugee girls in intergenerational sexual relationships. These categories were grouped under three themes, namely: age, gender inequality and low socio-economic status. Conclusion: In order to reduce the involvement of young refugee girls in intergenerational sex which increases their risk of HIV infection, the study recommends the development and/or improvement of programmes using multispectral approach to attend to the growing situation.
\end{abstract}

Keywords: HIV/AIDS, intergenerational sex; poverty; refugee girls

\section{Introduction}

Human immunodeficiency virus (HIV) is still spreading despite all available programmes to combat it both internationally and in South Africa. The rising spread is related to different behaviours which fuel the spread of HIV. One of those behaviours is intergenerational sexual relationships. Intergenerational sexual relationships is defined as the sexual relationship where young women have sex with men who are 5 years or older than them (Doherty, Shiboski, Ellen, Adimora \& Padian, 2006). Authors like Gregson (2002) and Hope (2007) describe it as relationships between young women aged 15 to 19 who report having sex in the last 12 months with a man who is 10 years and more older than themselves.

Intergenerational sex is one of the key drivers of the Human Immunodeficiency Virus (HIV) and Acquired Immune Deficiency Syndrome (AIDS) epidemic (Chinake, Dunbar \& Van der Straten, 2002). Doherty et al (2006) views or identifies intergenerational sex as dissortative sex, which is sexual partnership between individuals from low HIV risk group and those from high HIV risk group. This is considered as dissortative sexual relationship because young people who have had less sexual exposure are sexually connected with adults, whose HIV infection rates are likely to be higher. Intergenerational relationship plays an important role in the observed differences in HIV epidemiological patterns among refugee girls and boys of the same age (Leclerc-Madlala, 2008). Even if the prevalence of intergenerational sexual relationships and motivations behind such relationships are contested, a study among young women and girls in South Africa confirms that young women and girls living in poorer households have a higher likelihood of having exchanged sex for money (Hallman, 2005).

Intergenerational sexual relationship is also practiced by young refugee girls to satisfy their socio-economic needs (Nkosana, 2004). Intergenerational sexual relationships, disruption of family, increased sexual violence and increase in socio-economic vulnerability all occur mainly as a result of poverty (Hallman, 2005). Intergenerational sexual relationships increase the HIV infection rate among young refugee girls (Shisana et al., 2009). Luke and Kurz (2003) mentioned that if 
young women appear to be willing to engage in intergenerational sexual relationships, it is because they are vulnerable to exploitation due to poverty. Other girls are drawn to the transactional nature of intergenerational sex to receive gifts, money or cell phones in exchange for sex (Poulin, 2005).

Girls and young women are sometimes accused of luring adult men into relationships in order to obtain status and gain access to the infamous 'four Cs: cash, cellphones, clothes and a cars, which are mentioned repeatedly in the United Nations International Children's Emergency Fund (UNICEF), (2003) report. The older men are often called 'sugar daddies" by refugee girls and women. "Sugar daddies" are older men with numerous previous sexual partners who expose young refugee women and girls to potential abuse, pregnancy and infectious diseases such as HIV infection (Mohga, 2002). Doherty et al (2006) mentioned that with intergenerational sex, the main contributing factors to the HIV spread is power imbalance, non-condom usage and high possibility of starting the relationship with HIV discordant results (one partner being HIV negative and the other HIV positive). Meel (2003) mentioned that sometimes, men who know that they are infected with HIV intentionally engage in sex with young women and girls with the belief that sex with virgin is a cure for HIV which is just a myth.

South Africa hosts a large number of refugees because of the porousness of its borders (Uwantege, 2007). Young refugee girls are engaged in sex with older men under the guise of the "sugar-daddy" phenomenon with the primary motive of obtaining economical favours. HIVIAIDS prevention messages do not always reach migrants as a result of language barriers and the disadvantage in terms of access to proper medical care and information (International Organization for Migration, 2010). Girls and young women in relationships with older men are even more at risk due to an increased lack of knowledge about HIV transmission (United Nations Development Programme, 2003).

\section{Method}

\subsection{Aim}

This paper reports on a study that explored factors contributing to young refugee girls (aged 15-19 years) becoming engaged in intergenerational sexual relationships in the City of Tshwane, South Africa.

\subsection{Design}

In general, and though several studies have been conducted on factors contributing to intergenerational sex, minimal empirical attention has been directed towards examining the factors that contribute to this phenomenon among refugee girls in South Africa. This prompted the researchers to follow a phenomenological approach. Phenomenological methods are considered effective for studying under researched and poorly understood phenomena (Smith, Flowers \& Larkin, 2009). Interpretative phenomenological analysis (IPA) was utilised as a method and a tool for analysis. This approach was selected as the researcher wanted to offer expert analysis of the factors contributing to intergenerational sex among young refugee girls living in the City of Tshwane.

\subsection{Sampling and participants}

The study was conducted in Tshwane, Pretoria the capital city of South Africa. The city of Tshwane was purposively selected for accessibility and because one of the researchers is a refugee in the city which makes it easier to access young refugee girls involved in intergenerational sexual relationships. Following ethical clearance and management permission to conduct the study, participants were identified and recruited. Purposive and snowballing techniques were used to select the participants. One of the researchers knew two refugee girls who are involved in intergenerational sexual relationships. These girls also lead the researchers to other refugee girls who are involved in intergenerational sexual relationships. The study sample consisted of 20 refugee girls from different African countries (Democratic republic of Congo (four), Kenya (two), Nigeria (four), Rwanda (two), Tanzania (two) and Zimbabwe (six). The number was determined by data saturation. The ages of participants ranged between 18-19 years intentionally excluding those younger than 18 years owing to the challenges of obtaining consent because most of them were not living with their parents, and those who had parents, did not want them to know what they are involved in.

\subsection{Data collection and analysis}

Consent was sought and obtained from each participant before data collection. Data were collected between April and 
May 2013. In-depth individual interviews were conducted with each participant in order to collect first-hand information. The session started with questions on a variety of informal, conversational aspects about being refugees in South Africa in order to gain trust and make the participants more comfortable. This was followed by central question: "What make you get involved into a relationship with an older man?" Probes, minimal encouragers and follow-up questions were used to enhance the richness of responses. The interviews were audio-taped and each interview lasted about 45 to 60 minutes. Field notes were compiled to capture and describe the non-verbal cues observed during the interviews. At the end of each interview, some time was spent on debriefing the participants and referring them to relevant areas of help such as a counselling centre, social work depending on the seriousness of the socio-economic situation.

The audio-recorded interviews were transcribed verbatim into textual materials that were manually and independently analysed by two researchers using Smith`s (2005) IPA approach to analysis. Analysis proceeded parallel with the interviews, and was done repeatedly throughout the interview period until category saturation was achieved. Analysis was carried out in stages for each transcript. The analyses consisted of reading and re-reading transcripts, making notes and developing emergent themes. Connection between emergent themes was explored and related themes were clustered, resulting in the development of major themes. The outcome of this approach further resulted in a master list of themes for each transcript containing themes, sub-themes and associated quotes to illustrate where in the transcripts these themes could be found. The master lists of transcripts were compared and similarities were examined. The outcomes thereof were separate master lists of themes.

\subsection{Rigour}

Rigour is a measure of the overall quality of research, reflected in the data collection and analysis processes (Macnee \& McCabe, 2008). This study adopted Guba and Lincoln's (1994) framework of trustworthiness, as it fits in well with phenomenological studies. It includes five criteria, namely credibility, dependability, conformability, transferability and authenticity. Only some of these are referred to here. Interviews were audio recorded and field notes were taken during interviews to ensure dependability and conformability. A sample of the audio recorded data were independently transcribed and analysed by the researchers also for conformability. Both researchers agreed on the outcome of their analyses. The transcribed interviews were returned to the participants to verify the content and accuracy (member checking and validity checks) to enhance credibility and dependability of the study outcomes. Validity checks on the master list of themes were carried out by an independent researcher to ensure that the themes were relevant and evidenced in the data.

\subsection{Ethical Measures}

Approval to undertake the study was obtained from the Ethics Research Committee of the University of South Africa and the Tshwane City Municipality. All interviews were conducted in a secure private place to ensure privacy. Informed consent was obtained from all the participants, and anonymity was maintained using pseudonyms. Follow-ups, debriefing and even referrals were done according to needs of participants after the interviews.

\section{Results}

Three major super-ordinate themes emerged from data analysis: age, gender inequality and socio-economic status. Each super-ordinate theme has sub-themes. A short description of the findings is presented with and illustrated by excerpts from participants' narratives to enhance understanding of discussions presented

\subsection{Age}

As revealed in the findings of this study, age is one of the major factors that contributed to engagement in intergenerational sexual relationships as a result of powerlessness, increased libido and curiosity.

\subsubsection{Powerlessness}

Refugee girls are coerced to engage in intergenerational sexual relationships with older men irrespective of age discrepancy. Their ability to resist engaging in such relationship is mostly limited particularly when their families support the idea for financial or material gain. Since some of the older men are their fathers' age, they are most likely, 
psychologically, compelled to respect their elders' views and, therefore, consent to engage in relationships. Hence, they feel more secure and supported in relationships with older men than with men of their age. Statements like:

"He is much older than me. I feel it is not easy to say no to what he says..."

"This man has the age of my father. When he speaks, I am unable to say no"

This leads one to believe that refugee girls really struggle with a complex decision process leading up to their engagement in intergenerational sexual relationship encounters.

Since the fledgling age of these girls decreases their ability to insist on safe sex during intercourse, age must be viewed more as an impacting factor with regards to their vulnerability in intergenerational sexual relationships. It would appear that their young age puts them in an inferior position and makes them feel powerless to refuse such relationships and negotiate safer sex.

"It was difficult for me to insist on the use of condoms when I was dealing with my partner who was very older than me because I felt inferior."

By and large, age is a contributing factor, because a young age is typically associated with overall lack of experience and capacity to make informed decisions. In addition, the partners of refugee girls who are more astute can easily persuade them to abandon their initial prudent stance. These girls do not really have a lot of experience to help them negotiate the demands of older men who, most of the time, are more experienced in sexual issues.

"It was difficult for me to make decisions on sexual issues with my boyfriend because he used to say that I am younger than him and I do not know anything about sex."

Age differences causes refugee girls to be more at risk of contracting sexually transmitted infections including HIV. This is associated with feelings of being disempowered despite the knowledge of the sexual risky behaviours.

"Though I know that he has sexual relationship with other girls and there is a risk of HIV and other Sexual transmitted infection contraction, I feel disempowered to take a firm decision."

Hence, it appears that refugee girls face difficulties in negotiating sexual relationships, because their age makes them feel inferior to their much older partners.

Another notable aspect of powerlessness of refugee girls and women who are engaged in intergenerational sexual relationships is fear of the absence of support. In refugee situations, the move to a new community; not knowing the do's and don'ts; authority figures to report to; how things work are all complex interacting concerns that exacerbate powerlessness in these refugee girls.

\subsubsection{Increased Sexual Libido}

Teen age is also associated with high libido, which results in refugee girls seeking all kinds of sexual relationships, including intergenerational sexual relationships. The statement of one participant regarding the reasons for her involvement in sexual relationships confirms the above analysis:

"I used to have sex with my partner not only because of money and gift but because most of the time I feel the desire to have sex. This desire pushes me to have sex with anyone who can invite me to have sex with them".

This goes to say that refugee girls can also be exposed to intergenerational sexual relationships and the risk of HIV infection due to the fact that their age is associated with heightened sexual urges.

\subsubsection{Curiosity}

Curiosity is the desire to explore everything, including sexual experiences. This also exposes refugee girls to any type of sexual activities including involvement in intergenerational sexual relationships. This is more of the case when they are exposed to such sexual information through different means of communication as indicated by the following excerpt:

"I got involved in sexual activities to experiment what I heard from friends, saw on television, and heard on radio about 
sex and sugar daddies. So, I also felt the need to practice it in my life".

Thus, to experiment and discover sexual fulfilment and the benefits that intergenerational sexual relationships offer, make refugee girls to become involved in a variety of sexual relationships and run the risk of contracting HIV infection.

\subsection{Gender Inequality}

Apart from age, gender inequalities also significantly contribute to the engagement of refugee girls in intergenerational sexual relationships. As discussed earlier, gender inequality, which, by every means, still prevails in contemporary society also renders refugee girls vulnerable to intergenerational sexual relationships. Gender inequality refers to power differentials based on sexual identity between sex partners, and plays an important role in influencing decision-making in relationships.

\subsubsection{Inferior Status of Women}

Women are generally considered inferior to men in the majority of societies in the world. This is the same way refugee girls are treated compared to refugee boys. Refugee girls feel disempowered when having to make decisions because of the prevailing position which attributes more power to men than women. One participant stated:

"It was very difficult, because he used to say that he is the man and the last word and taking decisions belongs to him as a man. This pushed me to accept everything that he said to keep him with me".

To this point, much can be said about gender inequality which reduces the ability of refugee girls' to negotiate safer sex with their partners or even refuse intergenerational sexual relationships. It seems that older men are in fact unlikely to tolerate refusals from girls. Again, this exposes refugee girls to the risk of HIV infection.

The way refugee girls respect their gender associated status also affects their decision regarding sexual health information as illustrated by the following quote:

"I cannot seek information about HIV prevention or sex from the clinic as a woman because people will think that I am promiscuous. This makes it difficult for me to discuss anything about safe sex".

Indeed, the lack of information about HIV prevention compounded by gender norms exposes refugee girls involved in intergenerational sexual relationships to sexual manipulation and HIV infection.

\subsubsection{Attitudes towards Assertive Women}

Apart from their reluctance of attaining safer sex information, refugee girls are unable to introduce condom usage in relationships in fear of being accused of having extramarital relationships and being disrespectful to the cultural norms. One respondent related her experience as follows:

"I use to go to him with the condoms in my handbag but I was afraid to show him because I was afraid that he will say I am promiscuous".

\subsubsection{Cultural Believes}

Dominant African cultural norms which prescribe unconditional respect and obedience to a male partner also contribute to the involvement of refugee girls in intergenerational sexual relationships as they are unable to deny sexual demands by such men. It also increases the HIV infection risk as they cannot challenge any sexual practices as mentioned in this quotation:

"I was supposed to accept all propositions from my partner because he is very much older than me. My culture taught me to respect my husband and older people at all cost. As a result, it was difficult to refuse any of my partner's demands".

According to some African cultures, girls are still regarded by men as objects of pleasure who should not refuse 
their sexual advances. This culture enables men to sprawl their sexual network including their spouses, casual girlfriends and adolescent girls. One respondent on this issue said:

"I don't have control as my partner told me that according to his culture man can have sex with his partner anytime 'he' wants. If I refuse, he will seek sexual satisfaction outside the relationship and it will be my fault."

What this strongly implies is that girls are bound to become involved in intergenerational sexual relationships, because men think that they are entitled to a woman's body. They do not imagine that a woman has any right to refuse their demands for sex.

One would think that an older man, regarded as a father figure will help refugee girls to deal with some of the hardships of life. Instead, they often abuse their age and position to manipulate refugee girls into having risky sexual activities Thus, it is explicitly accepted that men would play decision- making and leadership roles in society, while women would play subservient, supporting and nurturing roles. One respondent reported that:

"Older men provide affection, protection and they know how to take care of me as compared to young men. That is why I support my culture which says that it is better to have a man who is older than you because he will be able to protect you".

The fact that women have been nurtured to believe that a relationship with an older man will provide protection makes them vulnerable.

\subsection{Socio-Economic Conditions}

This theme provides a deeper understanding of how socio-economic factors render refugee girls vulnerable to intergenerational sexual relationships. The sub-themes discussed under socio-economic factors include anonymity, high cost of living, unemployment, poor educational status and residential status

\subsubsection{Anonymity}

Anonymity associated with living in big cities also contributes to intergenerational sexual relationships. Sexual practices of this nature would likely be objectionable in rural areas or less crowded towns, but it is considered normal in a big city context as both partners feel anonymous. This is even facilitated in instances where refugee girls rent their own rooms and live there without parental supervision. This results in their involvement in intergenerational sexual relationships without any fear of discrimination. One of the participants said:

"I feel free to live with any man including older men in this city as the city is very big and no one can control my life or disturb me. In fact I do not even know who my neighbours are or what they are doing. Everyone mind their own business."

\subsubsection{High Cost of Living}

Living conditions in big cities such as the City of Tshwane, Pretoria is costly. Because of the high cost of living, refugee girls who live in the City believe that the only way of dealing with this situation is to become involved in a sexual relationship with older men who can provide for them to meet their economic needs. One of the participants stated that:

"Everything is so expensive in this city. My partner is the one who takes care of me and who pays my accommodation, so I cannot object to his sexual request".

Indeed, this is one of the main factors exposing refugee girls in urban areas to a higher HIV infection than those in rural or semi-urban areas.

\subsubsection{Unemployment}

Unemployment also contributes to the vulnerability of refugee girls to intergenerational sexual relationships. Chances of refugee girls getting employed are very limited to none due to lack of qualifications, experience and necessary legal documentations such as identity documents and work permits. This propels refugee girls to engage in transactional sex to 
meet their basic needs despite the possible devastating consequences. A respondent stated that:

"To find a job in this country (South Africa) is very difficult. Due to a lack of employment, I have a 'sugar daddy' who gives me money to help me provide for my needs."

Unemployment further increases the chances of refugee girls becoming involved not only in mono-androus (one female related to one man) intergenerational sexual relationships, but to poly-androus intergenerational relationship in order to get adequate money. This situation in turn makes them dependent on men for physical and economic security. One respondent said:

"I was living without any support because no one was able to help me. So to survive I did put myself into sexual relationship with several older men. Using condoms in such relationship was very difficult for me in fear of losing the support. As a result, I fell pregnant, another burden now."

Indeed, it seems that when refugee girls are unemployed they seek older men who can provide and meet their needs. This type of relationship is accompanied by a high risk of contracting HIV and other sexually transmitted infections (STIs). Such unstable unemployment circumstances expose and increase the vulnerability of women to HIV.

\subsubsection{Low Educational Status}

Poverty is a primary reason why many refugee girls could not complete their education, contributing to their vulnerability. When refugee girls are deprived of education, they are more likely to become involved in intergenerational sexual relationships. A lack of education reduces a young girl's ability to understand and practice HIV prevention techniques. One participant said:

"I did not attend school due to lack of money to pay my school fees. This is the reason why I entered into sexual relationships with the older man who is buying me food and paying for my accommodation. I cannot say anything due to lack of knowledge about sex with my partner who is a director".

Refugee girls seem to be unable to discuss sexual matters with their partners due to a lack of knowledge in comparison to their partners. This is due to limited schooling that would have provided some health education regarding the prevention of HIV. Of the total number of participants, only three completed high school, while others did not go beyond primary school-level education. Since the majority of these girls are now left with a sub-standard education their behaviours are affected, specifically their sexual behaviours.

The lack of appropriate education makes refugee girls vulnerable to a myriad of sexual issues including intergenerational sex. This study found that refugee girls who did not progress in school were more likely to become involved in sexual relationships with older men. In addition, these girls did not perceive any risks of HIV infection associated with this type of relationship. As indicated by one respondent who said:

"I prefer my 'sugar daddy' because he can take good care of me. When I need something, he can give it to me and I am doing this because I was unable to go to school, and there is no guarantee getting a job to support me".

Once again, as emphasised in other aspects on this study's findings, a lack of education limits the amount of information that people receive with regard to health and HIV prevention. Their low educational level exposes them to the high risk of HIV infection. A lack of education disempowers refugee girls from negotiating safe sex practices in intergenerational sexual relationships. Information on the prevention of HIV nowadays is also provided at all formal school levels through life skills orientation and related studies. Even if these refugee girls could gain access to information on HIV prevention from the Health Department, they still lack the basic skills of reading and interpreting such information. One of the participants said:

"I used to accept all the propositions of my partner. There is nothing new I can tell him and I feel like my argument is always weaker compared to his when it comes to the use of protective measures during sexual intercourse".

Education and knowledge regarding HIV infection is limited among refugee girls due to their lack of formal school education and access to information on HIV prevention. This is a serious problem when they engage in unprotected sex, since there is a high probability of HIV infection. 


\subsubsection{Residential Status}

Of all the participants in this study, five refugee girls had suitable accommodation, and were living with their parents. Two refugee girls lived on the streets without accommodation, while three others lived under pressure in 'HOP' accommodation (i.e. moving and unstable). This means that majority of the girls have accommodation that is being paid for with money from their sexual partners. The refugee girls who lived with their parents also contributed to paying rent at home. It is such situations that really force refugee girls to become involved with an older man in hope of them taking responsibility for their needs. One of the refugee girls reported saying:

\footnotetext{
"I got involved in a sexual relation with an older man because my parents do not have money. The money that I receive from my partner is used to pay rent."

"It is very difficult to live in any flat without money for the rent". That is why I decided to have a sugar daddy who pays my rent."
}

There is ample evidence that refugee girls become involved in intergenerational sexual relationships to obtain money for their families' rent and other needs. It is without doubt that some parents encourage intergenerational sexual relationships for their young daughters as a means of providing for their families.

The lack of accommodation makes refugee girls who are living in informal accommodations more vulnerable to sexual abuse 'This situation forces them into sexual violence at the hands of other refugee men, especially older men. This, in turn, exposes them to unwanted sexual intercourse, pregnancy, and HIV infection. One of the participants said:

"I was forced to join a group of refugees who were sleeping on the street because of lack of money to pay accommodation. So one day the leader of the place where we were sleeping promised to protect me, little did I realise that he was in fact the one who was sexually exploiting me because I was dependent on him".

\section{Discussion}

Intergenerational sexual relationship is rife among refugee girls in the city of Tshwane. The findings indicate that age disparity plays a major role in the involvement of refugee girls into sexual relationship with older men. Since they are much younger than their partners, they are likely to have less decision-making power as to when and how to have sex. Luke and Kurz (2002) argue that because of the limited negotiating power of refugee girls regarding sexuality and reproduction, sexual partnerships between refugee girls and older men are fundamentally imbalanced, with men having more power. The power imbalance that may exist between the person providing the money and the person receiving the money facilitates HIV transmission, since partners are not selected on the basis or criteria other than money (Muula, 2008).

Apart from powerlessness related to age difference, young girls have increased sexual desire by virtue of being teenagers. Literature reviews confirmed that with the attainment of puberty, hormonal changes occur, and the production of androgens in young people of both sexes, in particular, results in an increased sex drive (Berk, 2012). In response, adolescents become very concerned with managing sexual urges and sexuality in social relationships. Refugee girls have more raging sex hormones than older women and men. This influences their sexual behaviour, as they experience great and sometimes uncontrollable sexual urges with a strong desire to have sex more frequently than older women. The need to satisfy their sexual desire was also reported by Nkosana and Rosenthal (2007) who stated that the relationship between refugee girls and older men is associated with desire for pleasure and enjoyment by the younger partner. Therefore, the risk of exposure to HIV infection increases as opportunities for sexual encounters increases.

The refugee girls are at the age of wanting to explore. They are also at a stage of wanting to practically prove whatever information they get from any source apart from what is said by parents. This attests to Berk, (2012) who reported that some people are concerned that providing information about sex and sexuality arouses curiosity and can lead to sexual experimentation urges. Exploration also includes exploring sex with older men despite warning information related to risk of contracting HIV infection. In other situations it also involves intentional unprotected sex just to explore how sex without condom feels like.

Gender inequality was also identified as a contributing factor for young women to be involved in intergenerational sex. By virtue of being women, young girls are considered inferior compared to their male counterparts. The way in which boys of the same age with girls are treated in the community is different. Boys can openly discuss sexual issues without being prejudiced compared to girls of same age. Gender inequality may prevent refugee girls from revealing their knowledge of sexual matters, including the use of HIV prevention strategies which exposes them to HIV infection (Khan, 
2011). The relegation of women to a lower status and value due to gender norms, customs and laws increase the involvement of women in sexual risky behaviour with subsequent vulnerability to contracting HIV. Gender issues are at the core of many countries HIV epidemic which is fuelled by specific sexual behavior in which women often have little or no decision-making power (Betron et al., 2014). Gender norms related to sex can have detrimental effects on young women particularly in terms of STIs and HIV as well as the risk of sexual and physical violence (Khan 2011). Gender inequality between partners decreases the likelihood of refugee girls successfully negotiating condom use during sexual intercourse. The prevailing view of masculinity perpetuates the belief that men need frequent sexual gratification and multiple partners including sex with refugee girls. Mulumeoderhwa (2007)

Coupled with gender issues, there are also cultural beliefs that an older man is better suited for taking care of a young girl and protecting her and this perception results in refugee girls living with older men for protection and material, financial or physical gain (Leclerc-Madlala, 2008). According to some African traditions and cultures, women are expected to assume the roles of mothers and wives, fulfilling the sexual desires of men. They are also expected to be obedient and subservient to men who are regarded as protectors, guides, supervisors and leaders (Leclerc-Madlala, 2008:19). These cultural aspects prevent refugee girls from introducing condom usage in relationships in fear of being accused of being disrespectful to the cultural norms (Halperin \& Epstein, 2007). All these factors expose refugee girls to the risk of HIV infection.

Socio-economic status seems to be the major factor compelling young refugee girls to engage in intergenerational sex. The results clearly show that abject poverty influences the sexual behaviour of refugee girls. The conditions of poverty under which they live hinders them from discussing safer sex practices with their partners during sexual intercourse in fear of losing the economic support from their ' sugar daddies'. Several authors also attest that poverty is an important driving factor in unsafe sexual practices and in intergenerational sexual relationships (Madise et al., 2007; Onwuliri \& Jolayemi 2006). The unequal power relations reflected in such relationships affect girls' ability to refuse unsafe sex, and expose them to sexually transmitted infections such as HIV (The International Council of AIDS Service Organizations (ICASO), 2007; Mandela, 2002).

Leclerc-Madlala (2008) examined the factors that contributed towards protecting young women against their involvement with older men. They found that a combination of personal, social and institutional factors (that is, strong sense of self-worth, knowledge of sexual risks, acceptance of socio-economic circumstances, social support and religious values) helped refugee girls to successfully resist sexual advances from older men. Indeed, the researcher believes that giving refugee girls access to school education, as well as accommodation and knowledge about HIV prevention is very helpful. This invariably enhances their self-reflection and esteem, and empowers them in their sexual relationship decisions that is often characterised by coercion and manipulation on the part of older man.

\section{Conclusion and Recommendations}

The findings revealed that the main reasons for refugee girls getting involved in intergenerational sexual relationships is primarily due to socio-economic factors embodying the lack of necessary material and financial support from family, parents and organisations that have failed to provide them with HIV prevention information. This is further intensified by the inadequacy of funds and poor structure of organisations supporting refugees. Age, gender inequality and low socioeconomic factors increase sexual vulnerability when refugee girls are involved in intergenerational sexual relationships, because they are unable to insist on safe sex. It is evident that the conditions in which the refugee girls find themselves in cannot be addressed by one sector, but it needs a multi-sectoral approach to better assist in addressing the basic immediate critical ongoing needs of refugee girls, and this can be done at personal, community and structural levels.

\section{Limitation of the Study}

The researchers were faced with some constraints which have impacted on the results. Gaining access to information from refugee girls was not easy, because the research dealt with very sensitive information and issues. Participants were uncomfortable in responding to some questions posed to them. Language barrier was also a problem and in some cases communication or conversation was limited during the interview, but eventually translated for better understanding.

\section{Acknowledgements}

The authors are grateful to all refugee girls who took part in this study. The authors are also grateful to the persons who 
assisted with interpretation during interviews.

\section{References}

Berk, L. E. (2012). Child development. (9th ed.). United States of America: Pearson.

Betron, A., Barker, G., Contreras, J. E. \& Peacock, D. (2014). Men, Masculinities and HIVIAIDS: strategies for action. [Online] Available: http://menengage.org/wp-content/uploads/2014/04/Men-and-Boys_Final_Web.pdf, (May 2, 2014).

Chinake, H., Dunbar, M., \& Van der Straten, A. (2002). "Intergenerational sex among adolescents in Zimbabwe." Abstract no. MoOrE1116. XIV International Conference on AIDS, Barcelona, 7-12 July.

Doherty, I. A., Shiboski, S. Ellen, J. M., Adimora, A. A., Padian, N.S. (2006). Sexual bridging socially and over time: a simulation model exploring the relative effects of mixing and concurrency. Sexually Transmitted Diseases. 33 (6), 368-73.

Gregson, S., Nyamukapa, C. A., Garnett, G. P., Manson, P. R., Zhuwau, T., Carael, M. et al. (2002). Sexual mixing patterns and sexdifferentials in teenage exposure to HIV infection in rural Zimbabwe. Lancet, 359, 1896-1903.

Guba, E. G. \& Lincoln, Y. S. (1994). Competing paridigm in Qualitative Research. In Denzil, K.D \& Lincoln, Y.S. Handbook of Qualitative research, Thousands Oaks, CA: Sage Publications.

Hallman, K. (2005). Gendered Socioeconomic Conditions and HIV Risk Behaviours among Young People in South Africa, African Journal of AIDS Research, 4 (1), 37-50.

Halperin, D. T. \& Epstein, H. (2007). Why is HIV Prevalence so Severe in Southern Africa? The role of multiple concurrent partnerships and lack of male circumcision: Implications for AIDS prevention. The South African Journal of HIV Medicine. 19-25.

Hope, R. (2007). Addressing cross-generational sex. A desk review of research and programs. Population Reference Bureau. United State of America.

The International Council of AIDS Service Organizations (2007). Gender, Sexuality, Rights and HIV; An overview for community sector organizations. Toronto: ICASO

International Organization for Migration (2010). Migration and health in South Africa: A review of the current situation and recommendations for achieving the World Health Assembly Resolution on the Health of Migrants. IOM Regional Office for Southern Africa, Pretoria: South Africa.

Khan, A. (2011). Gender-based Violence and HIV: A Program Guide for Integrating Gender-based Violence Prevention and Response in PEPFAR Programs. Arlington, VA: USAID's AIDS Support and Technical Assistance Resources, AIDSTAR-One, Task Order 1.

Leclerc-Madlala, S. (2008). Age-disparate and intergenerational sex in southern Africa: The dynamics of hyper vulnerability. 22 (4), 1725.

Luke, N. \& K. Kurz. (2002). Cross-generational and Transactional Sexual Relations in sub-Saharan Africa: Prevalence of Behaviour and Implications for Negotiating Safer Sexual Practices. Washington, DC: ICRW/PSI.

Macnee, C. L. \& McCabe, S. (2008). Understanding Nursing Research. Reading and using research: Lippincott Williams. Philadelphia.

Madise, N., Zulu, E. \& Ciera, J. (2007). Is poverty a driver for risky sexual behaviour? Evidence from national surveys of adolescents in four African countries. African Journal of Reproductive Health. 11(3), 83-98.

Mohga, M. S. (2002). Gender, poverty, and intergenerational vulnerability to HIVIAIDS, Gender \& Development. 10 (3), 63-70.

Mandela, N. (2002) Closing speech, International Conference on HIV/AIDS, Barcelona.

Muula. A. S. (2007). Circumcision as HIV Prevention. Focus. A guide to AIDS prevention, 22 (6), 1-4.

Meel, B. L. (2003). The myth of child rape as a cure for HIVIAIDS in Transkei: a case report. Med Sci Law. 43, 85-8.

Nkosana, M. J. (2004). Intergenerational sexual relationships in urban Botswana, A thesis submitted in fulfilment of the requirements of the degree of Doctor of Philosophy, The University of Melbourne, Australia.

Nkosana, J. \& Rosenthal, D. (2007). The dynamics of intergenerational sexual relationships: the experience of schoolgirls in Botswana. Sex Health. 4,181-7.

Poulin M. (2007). Sex, money, and premarital partnerships in southern Malawi. Soc Sci Med. 65, 2383-93.

Poulin, M. (2005). Giving and Getting: Rethinking Sex, Money, and Agency among Youth in Rural Malawi. Paper presented at the Princeton Institute for International and Regional Studies Graduate Student Conference, April 8-9, Princeton, NJ, USA.

Shisana, O., Rehle, T., Simbayi, L.C., Zuma, K., Jooste, S., Pillay-van Wyk, V., Mbelle. N., Van Zyl, J., Parker, W., Zungu, N.P., Pezi, S. and the SABSSM III Implementation Team (2009). South African national HIV prevalence. Incidence and communication survey 2008: A turning tide among teenagers? Cape Town: HSRC Press.

Smith, J. A. (2005) Semi-structured interviewing and qualitative analysis. In, Smith JA, Harre R and van Langenhove L. (eds) Rethinking methods in psychology. London: Sage.

Smith, J. A., Flowers, P. \& Larkin, M. (2009). Interpretive phenomenological analysis: theory, method and research. London: Sage.

United Nations Development Programme (2003). Zimbabwe Human Development Report, Redirecting Our Responses to HIV and AIDS. New York: United Nations Development.

Uwantege, C. (2007). Refugee women, gender-based challenges, HIV and AIDS and the French-Swahili church in Pietermaritzburg. University of Kwazulu Natal. Durban. 